Conclusion Each year an increasing number of scans are performed, with good overall success rates; although a proportion of scans completed will meet 'invalid' criteria and this must be taken into account when interrupting the predicted fibrosis score. Inter-operator variability is evident and relates to prior formal training (undertaken by EchoSens) and total number of scans performed. Nurses undertake scans in dedicated clinics with a set time allowance and this may explain their higher success rates. Transient elastography should, therefore, be performed by those with formal training, undertaking regular scans in a dedicated clinic, to increase validity of results.

Disclosure of Interest None Declared.

\section{PWE-151 COMPARISON OF TYPE 1 AUTOIMMUNE HEPATITIS PATIENTS' CHARACTERISTIC IN CAUCASIAN, ASIAN AND BLACK ETHNIC GROUPS: A SINGLE CENTRE EXPERIENCE}

${ }^{1}$ NN Than* ${ }^{*}{ }^{2} J$ Mann, ${ }^{3} \mathrm{R}$ Gupta, ${ }^{3}$ J Hodson, ${ }^{3}$ P Nightingale, ${ }^{4}$ D Adams, ${ }^{1} Y H$ Oo. ${ }^{1}$ Centre for Liver Research and NIHR BRU, University of Birmingham and University Hospital Birmingham NHS Trust, Birmingham, UK; ${ }^{2}$ Leeds Royal Infirmary Hospital, Leeds, UK; ${ }^{3}$ Liver Unit, University Hospital Birmingham NHS Trust, UK; ${ }^{4}$ Centre for Liver Research and NIHR BRU, University of Birmingham and University Hospital Birmingham NHS Trust, Birmingham, UK

\subsection{6/gutjnl-2014-307263.411}

Introduction Autoimmune hepatitis (AIH) is a disease of unknown aetiology characterised by interface hepatitis, hypergammaglobulinaemia and circulating autoantibodies. ${ }^{1,2}$ It is associated with Human leucocyte antigen (HLA) DR3/DR4 allotypes which are common in European Caucasian population. ${ }^{2}$ Previous published studies reported late clinical presentations and poor outcome in non-Caucasian ethnic groups.

Methods This is a retrospective analysis of patients with type 1 $\mathrm{AIH}$ at a single centre tertiary liver transplant unit between year 1995 and 2012. Patients with simplified AIH score of more than or equal to 6 were included in the study. Data were collected thoroughly from electronic case notes, clinical letters and treatment charts. Basic demographics, clinical presentations, blood parameters such as biochemistry and immunology, liver histology and presence of other associated autoimmune conditions were documented. Those factors were compared among three ethnic groups: Caucasian, Asian and Black-African.

Results A total of 190 patients are included in the study. The majority $(78 \%)$ of $\mathrm{AIH}$ patients are females. Majority (84\%) were Caucasian and Asian ethnicity constitutes $12 \%$ of the study population with the remainder (4\%) being Black ethnicity. Age of diagnosis is slightly higher among Caucasian groups although non-significantly (Median age 50.5 vs 34.9 in Asian and 45.3 in Black).

No significant differences were detected for associated autoimmune conditions, DR3/DR4 association or liver biochemistry blood results among three ethnic groups. Immunoglobulin G and Immunoglobulin A are significantly lower in Caucasian compared to non-Caucasian populations $(\mathrm{p}=0.029,0.005$ respectively). There are no differences in clinical outcomes such as cirrhosis, development of hepatocellular carcinoma (HCC) or liver decompensation among three different ethnic groups.

Conclusion Clinical presentations and blood parameters were similar among three groups except Ig G and Ig A which were lower in Caucasian ethnic populations. Overall transplant free survival was similar among the three groups

\section{REFERENCES}

1 Zachou $\mathrm{K}$, et al. Review article: autoimmune hepatitis - current management and challenges. Alimentary Pharmacology and Therapeutics 2013. 38(8):887-913

2 Albert J, Czaja PTD. Genetic susceptibilities for immune expression and liver cell injury in autoimmune hepatitis. Immunological Reviews 2000;174:250-259

Disclosure of Interest

None Declared.

\section{PWE-152 PRIMARY SCLEROSING CHOLANGITIS-INFLAMMATORY BOWEL DISEASE IS ASSOCIATED WITH AN INCREASED FREQUENCY OF POST-TRANSPLANT COLONIC LYMPHOMA}

${ }^{1} \mathrm{PJ}$ Trivedi*, ${ }^{1} \mathrm{~T}$ Wright, ${ }^{1} \mathrm{~J}$ Robinson, ${ }^{1} \mathrm{~K}-\mathrm{K}$ Li, ${ }^{2} \mathrm{~B}$ Gunson, ${ }^{1} \mathrm{D}$ Adams, ${ }^{3} \mathrm{~J}$ Ferguson, ${ }^{1} \mathrm{G}$ Hirschfield. ${ }^{1}$ NIHR Biomedical Research Unit and Centre for Liver Research, UK; ${ }^{2}$ NIHR Biomedical Research Unit, University of Birmingham, UK; ${ }^{3}$ Liver Unit, University Hospitals Birmingham, Queen Elizabeth Hospital, Birmingham, UK

\subsection{6/gutjnl-2014-307263.412}

Introduction Post-transplant lymphoproliferative disease (PTLD) is a recognised complication of liver transplantation (LT). Although small intestinal involvement is common, factors predisposing to colonic disease are not well characterised.

Methods A case-note review of all patients undergoing liver transplantation (1982-2013) was performed, and subsequently cross-referenced with an institutional lymphoma database comprising all biopsy-proven PTLD cases to date. Putative risk factors for development of colonic lymphoma were analysed using SPSSv21.

Results Over a 31-year adult 'first liver' transplant experience (No. of recipients $=2872$ ), 72 cases of post-LT lymphoproliferative disease were identified and most commonly observed in the context of primary biliary cirrhosis $(\mathrm{PBC} ; \mathrm{n}=20$ ) and primary sclerosing cholangtitis (PSC; $\mathrm{n}=14$ ). Overall, intestinal involvement was observed in 18/72 patients, representing predominantly small bowel disease $(\mathrm{n}=12)$. Colonic lymphoma occurred only in individuals transplanted for PSC $(n=6$; median 5.0yrs post-transplant; IQR: 3.2-11.7), all of who had underlying colitis. In all cases, disease was a diffuse large B-cell lymphoma; however, only 2 patients had EBV-(LMP)-positive tumours, from which only 1 a detectable serum EBV-titre (qPCR). There were no significant associations with age at transplantation, male gender, treatment with azathioprine or tacrolimus, duration of azathioprine or calcineurin inhibitor exposure, or onset of colitis post-LT. Only one patient (each) with PTLD occurring in the context of cardiothoracic $(\mathrm{n}=6)$ and renal $(\mathrm{n}$ $=32$ ) transplantation developed large bowel disease, and under these circumstances was part of a disseminated lymphomatous process.

Conclusion PSC/colitis is associated with development of colonic lymphoma post-LT. Additional risk factors have yet to be identi, UKfied.

Disclosure of Interest None Declared.

\section{PWE-153 COST EFFECTIVENESS OF RIFAXIMIN-A IN THE REDUCTION OF RECURRENCE OF OVERT HEPATIC ENCEPHALOPATHY}

${ }^{1} \mathrm{CD}$ Poole, ${ }^{2} \mathrm{P}$ Conway* ${ }^{*}{ }^{3} \mathrm{~K}$ Nanuwa, ${ }^{2} \mathrm{~B}$ Joseph, ${ }^{1} \mathrm{C}$ Bannister, ${ }^{1} \mathrm{CJ}$ Currie. ${ }^{1}$ School of Medicine, Cardiff University, Cardiff, UK; ${ }^{2}$ Norgine Global Health Outcomes, Norgine Ltd, UK; ${ }^{3}$ Norgine UK, Uxbridge, UK

10.1136/gutjnl-2014-307263.413 